\title{
Publisher's Note: Translocation of a semiflexible polymer through a nanopore in the presence of attractive binding particles [Phys. Rev. E 92, 032711 (2015)]
}

Ramesh Adhikari and Aniket Bhattacharya

(Received 18 April 2016; published 27 April 2016)

DOI: 10.1103/PhysRevE.93.049907

This paper was published online on 14 September 2015 with errors throughout the text of the article and in Fig. 9. There are also formatting errors in Eqs. (2), (5), and (6). In the following locations, $\rho^{0.8}$ should read as $\rho^{-0.8}$ : in the abstract, in Fig. 9 (revised figure provided below), in the caption to Fig. 9, and in the last line of the first paragraph on page 7. Equation (2) should read as

$$
\begin{aligned}
U_{\mathrm{LJ}}(r) & =4 \epsilon\left[\left(\frac{\sigma}{r}\right)^{12}-\left(\frac{\sigma}{r}\right)^{6}\right]+\epsilon \text { for } r \leqslant 2^{1 / 6} \sigma \\
& =0 \text { for } r>2^{1 / 6} \sigma .
\end{aligned}
$$

Equation (5) should read as

$$
\begin{aligned}
U_{\text {binding }}(r) & =4 \epsilon_{c}\left[\left(\frac{\sigma}{r}\right)^{12}-\left(\frac{\sigma}{r}\right)^{6}\right]+\epsilon \text { for } r \leqslant 2.5 \sigma \\
& =0 \text { for } r>2.5 \sigma .
\end{aligned}
$$

Equation (6) should read as

$$
\begin{aligned}
U_{\mathrm{W}}(r) & =4 \epsilon_{c}\left[\left(\frac{\sigma}{r}\right)^{12}\right]+\epsilon \text { for } r \leqslant 2^{1 / 6} \sigma \\
& =0 \text { for } r>2^{1 / 6} \sigma .
\end{aligned}
$$

The paper has been corrected as of 18 March 2016. The text, equations and figure are incorrect in the printed version of the journal, therefore for the benefit of the print readership, Fig. 9 has been replicated below along with its caption.

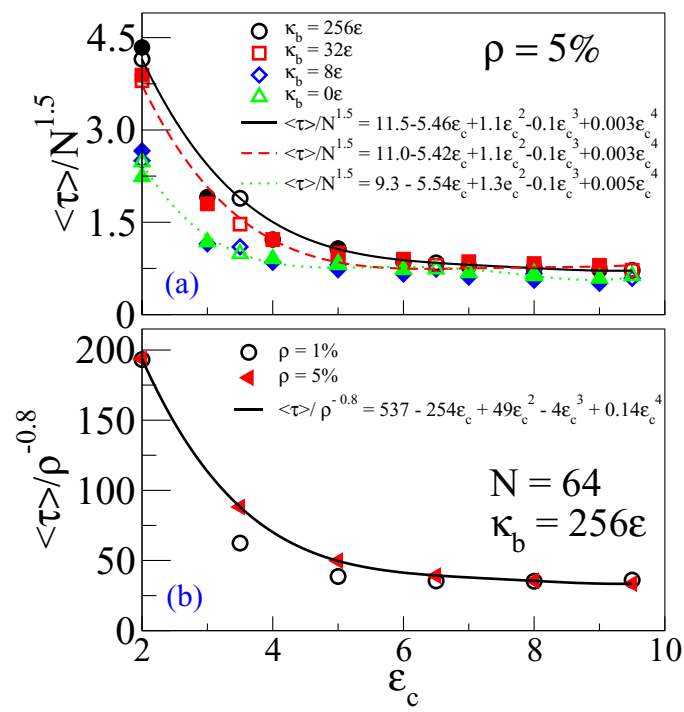

FIG. 9. (a) Scaled MFPT $\langle\tau\rangle / N^{1.5}$ as a function of binding strength $\epsilon_{c}$ of BPs for different chain stiffness $\left(\kappa_{b}=0,8 \epsilon, 32 \epsilon\right.$, and $\left.256 \epsilon\right)$ and for $N=64$ (open symbols) and $N=32$ (closed symbols), respectively. Black-solid, red-dashed, and green-dotted lines are fourth-degree polynomial fits to the data for $\kappa_{b}=256 \epsilon, 32 \epsilon$, and $8 \epsilon$, respectively. The fitted line for the data corresponding to $\kappa_{b}=8 \epsilon$ is almost the same as for $\kappa_{b}=0 \epsilon$. (b) Scaled MFPT $\langle\tau\rangle / \rho^{-0.8}$ as a function of binding strength $\epsilon_{c}$ of BPs for a stiff chain $\left(\kappa_{b}=256 \epsilon\right)$ of length $N=64$. Open symbols correspond to $\rho=1 \%$ and closed symbols correspond to $\rho=5 \%$. The solid line represent the fourth-degree polynomial fit to the data. 